\title{
Synthesis and Structure of Organic-Inorganic Hybrid Semi-interpenetrating Polymer Network Gels
}

\author{
Naofumi Naga ${ }^{1}$, Yukie Uchiyama ${ }^{1}$, Yuri Takahashi ${ }^{1}$, Hidemitsu Furukawa ${ }^{2}$ \\ ${ }^{1}$ Department of Applied Chemistry, Materials Science Course, College of Engineering, Shibaura Institute of \\ Technology, 3-7-5 Toyosu, Koto-ku, Tokyo 135-8548, Japan \\ ${ }^{2}$ Department of Mechanical Systems Engineering, Graduate School of Science and Engineering, Yamagata University, \\ 4-3-16 Jonan, Yonezawa, Yamagata 992-8510, Japan \\ Correspondence: Naofumi Naga, Department of Applied Chemistry, Materials Science Course, College of Engineering, \\ Shibaura Institute of Technology, 3-7-5 Toyosu, Koto-ku, Tokyo 135-8548, Japan
}

Received: January 5, 2016 Accepted: January 7, 2016 Online Published: January 25, 2016

doi:10.5539/ijc.v8n1p165 URL: http://dx.doi.org/10.5539/ijc.v8n1p165

\begin{abstract}
Semi-interpenetrating polymer network (semi-IPN) gels have been synthesized using a hydrosilylation reaction of 1,3,5,7-tetramethylcyclotetrasiloxane (TMCTS) as a joint molecule, and $\alpha, \omega$-nonconjugated dienes, 1,5-hexadiene (HD) or 1,9-decadiene (DD) as linker molecules in the presence of polystyrene (PS) as a liner polymers in toluene or cyclohexane. Network structure, mesh size and mesh size distribution, of the resulting semi-IPN gels was quantitatively characterized by means of a scanning microscopic light scattering (SMILS). The relaxation peaks derived from three kinds of structures were detected in the semi-IPN gels prepared in toluene by the SMILS analysis. One was derived from the mesh formed by TMCTS/ $\alpha, \omega$-nonconjugated dienes about 1-2 nm. Others were derived from transition networks about 20-150 nm and large clustered liner polymer chains about 700-2300 nm. Effect of concentration and molecular weight of the liner polymer on the network structure of the semi-IPN gels in toluene was investigated. The relaxation peaks derived from transition networks or random coils formed by aggregated PS chains were detected in the semi-IPN gels containing high concentration or high molecular weight PS. The semi-IPN gels containing PS were also prepared in cyclohexane as a poor solvent for PS at $40^{\circ} \mathrm{C}$, which was a higher temperature than the upper critical solution temperature (UCST $=34^{\circ} \mathrm{C}$ ) of PS in cyclohexane. The network structure of the semi-IPN gels was traced by SMILS on the cooling process. In the semi-IPN gel with the short linker molecule of HD, the relaxation peak derived from clustered PS chains was detected over the UCST. By contrast, the relaxation peak derived from transition network was observed in the semi-IPN gel with the long linker molecule of DD.
\end{abstract}

Keywords: semi-interpenetrating gel, organic-inorganic hybrid gel, polystyrene

\section{Introduction}

Organic-inorganic hybrid polymers having network structures have been developed due to their characteristic properties: high transparency, high thermal stability, good mechanical strength, excellent solvent resistance, low dielectric constant, and so on. The organic-inorganic hybrid polymers have been prepared by some effective methods (Mark et al., 1995; Mascia, 1995; Novak, 1993; Provatas et al., 1997; Laine et al., 1998; Vaia et al., 1996; Sogah et al., 1999; Saegusa et al., 1991; Landry st al., 1993; Yamada et al., 1997; Chujo et al., 2003; Laine et al., 2003; Galiastsatos et al., 2001). Hydrosilylation reaction of multi-functionalized crosslinking reagents containing $\mathrm{Si}-\mathrm{H}$ or vinyl group is one of the effective methods to yield the organic-inorganic network polymers containing Si (Yoshida et al., 2004, 2001; Tsumura et al., 1998, 1999, 2000; Michalczyk et al., 1993; Lercher et al., 2002; Laine et al., 1998; Schaefer et al., 2003). The authors recently attained effective synthesis of organic-inorganic hybrid gels by means of a hydrosilylation reaction of cyclic siloxane or cubic silsesquioxane with $\alpha, \omega$-nonconjugated dienes. Characterization of the network structures of the gels was quantitatively investigated by a scanning microscopic light scattering (SMILS) system (Naga et al., 2006). The SMILS analysis of the gels from cyclic siloxane or cubic silsesquioxane with $\alpha, \omega$-nonconjugated dienes showed the extremely narrow distribution of mesh size in the gels. Further more, the mesh size of the gels could be controlled by the length of the $\alpha, \omega$-nonconjugated dienes used (Naga et al., 2006). We also reported co-gelation of siloxane or silsesquioxane and $\alpha, \omega$-nonconjugated dienes to control the mesh size of these organic-inorganic hybrid gels precisely (Naga et al., 2007). One of the next steps of this study should be modification and application development of the organic-inorganic hybrid gel. 
We focused on concept of interpenetrating polymer network (IPN) for the modification of the hybrid gel. The IPN is composed of two kinds of network structures which are entangled each other. The IPNs have been developed as one of the useful methods to compound the polymers which are immiscible. The IPN is also effective to add some functions and/or to improve its thermal and mechanical properties. Some kinds of gels have been developed based on the concept of the IPN. For example, a hydrogel prepared by in-site polymerization of acrylaminde within a crosslinked poly(2-acrylamide-2-methyl-1-propane sulfonic acid), so called "double network gel", which shows extraordinary strong mechanical properties (Gong, et al., 2003, 2004, 2005, 2007). We came up with an idea to synthesize the organic-inorganic hybrid gel in the presence of liner polymers, namely semi-IPN gel. Well-defined network structure of the present organic-inorganic gel should be useful to develop the semi-IPN gels with functionalities derived from the linear polymer. For a preliminary examination, we selected atactic-polystyrene (PS) as a linear polymer. PS is soluble in kinds of organic solvents which do not poise the Pt catalyst used to synthesize the present organic-inorganic hybrid gel. In this basic study, we conducted a hydrosilylation reaction of TMCTS with HD or DD in the presence of PS to form the semi-IPN gels in toluene, which is a good solvent for PS, as shown in Scheme 1. The semi-IPN gels with PS were also prepared in cyclohexane, which is a poor solvent for PS. The network structure of the resulting semi-IPN gels was investigated by SMILS to study the effects of the solvent, molecular length of dienes, concentration \& molecular weight of PS, or temperature on the network structure.<smiles>C[SiH]1O[SiH](C)O[SiH](C)O[SiH](C)O1</smiles>

TMCTS<smiles>CC(CC(C)(C)C)c1ccccc1</smiles>

PS

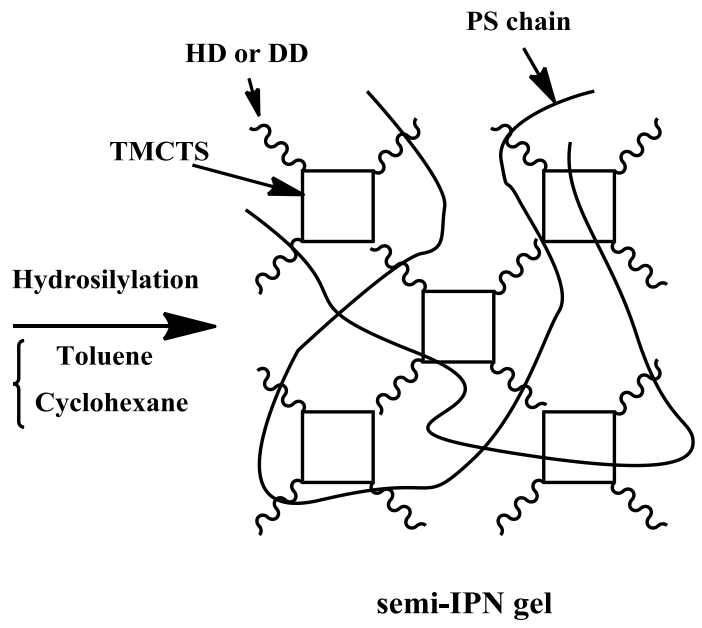

DD

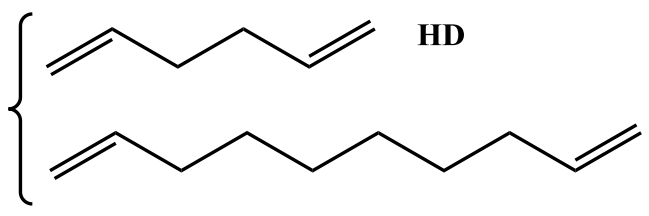

Scheme 1. Synthesis of organic-inorganic hybrid semi-IPN gels by means of a hydrosilylation reaction of TMCTS with $\mathrm{HD}$ or DD in the presence of PS.

\section{Method}

\subsection{Materials}

1,5-Hexadien (HD) and 1,9-decadiene (DD) (Tokyo Kasei Kogyo Co.) were dried over calcium hydride and distilled under nitrogen atmosphere before use. 1,3,5,7-Tetramethylcyclotetrasiloxane (TMCTS) (Chisso Co. Ltd.), illustrated in Scheme 1, were used without further purification. Platinum-divinyltetramethyldisiloxane complex (1) was purchased from Chisso Co. Ltd., and used without purification. Toluene and cyclohexane were dried over calcium hydride under refluxing for $6 \mathrm{~h}$ and distilled under nitrogen atmosphere before use. The platinum complex 1 was dissolved in distilled toluene or cyclohexane $(0.6 \mathrm{mM})$, and stored under nitrogen atmosphere. PS samples were synthesized by anionic polymerization of styrene with n-butyllithium in cyclohexane at room temperature. Molecular weight and molecular weight distribution, determined with gel-permeation chromatography, of the obtained PS samples are as follows, PS1: $M_{\mathrm{n}}=15,000, M_{\mathrm{w}} / M_{\mathrm{n}}=1.4$, PS $2: M_{\mathrm{n}}=69,000, M_{\mathrm{w}} / M_{\mathrm{n}}=1.2$, PS $3: M_{\mathrm{n}}=347,000, M_{\mathrm{w}} / M_{\mathrm{n}}=1.6$.

\subsection{Synthesis of the semi-IPN gels}

The molar ratio of vinyl group in $\alpha, \omega$-nonconjugated diene to Si-H group in TMCTS was adjusted to 1.0. Molar ratio of diene to catalyst 1 was $5000 \mathrm{~mol} / \mathrm{mol}$ in the reaction system.

\subsubsection{Synthesis of TMCTS-HD semi-IPN gel in toluene (12 wt $\%$ gel containing $5.0 \mathrm{wt} \%$ of PS1)}

In a sample tube of $4 \mathrm{~mm}$ diameter, PS1 $10.1 \mathrm{mg}$, TMCTS $14.4 \mathrm{mg}(0.06 \mathrm{mmol})$ and $\mathrm{HD} 9.9 \mathrm{mg}(0.12 \mathrm{mmol})$ were dissolved in a $155.0 \mu \mathrm{L}$ of toluene. Then a $40.0 \mu \mathrm{L}$ of toluene solution of the catalyst $1(0.6 \mathrm{mM})$ was added and mixed at room temperature. It was placed without stirring, and the whitely nebulous gel was generated presently. The semi-IPN gels with other PS concentrations and higher molecular weight PS (PS2) were also synthesized by the same 
procedure.

2.2.2 Synthesis of TMCTS-HD semi-IPN gel in cyclohexane (12 wt $\%$ gel containing $0.1 \mathrm{wt} \%$ of PS3)

In a sample tube of $4 \mathrm{~mm}$ diameter, TMCTS $14.4 \mathrm{mg}(0.06 \mathrm{mmol})$ and HD $9.9 \mathrm{mg}(0.12 \mathrm{mmol})$ were dissolved in a 129.6 $\mu \mathrm{L}$ of cycloheaxne, and a $42.4 \mu \mathrm{L}$ of $0.5 \mathrm{wt} \%$ cyclohexane solution of PS3 $(0.17 \mathrm{mg})$ was added at a fixed temperature $\left(28\right.$ or $\left.40^{\circ} \mathrm{C}\right)$. Then a $42.4 \mu \mathrm{L}$ of cyclohexane solution $(0.6 \mathrm{mM})$ of the catalyst 1 was added and mixed (TMCTS + HD $=12.0 \mathrm{wt} \%, \mathrm{PS}=0.1 \mathrm{wt} \%$ in the reaction system). It was placed without stirring, and the whitely nebulous gel was generated presently. Synthesis of the semi-IPN gel with DD (TMCTS + DD = 10.0 wt $\%$ ) was also synthesized by the same procedure.

\subsection{Measurements}

Quantitative determination of minute mesh size of the gels was performed with the scanning microscopic light scattering (SMILS) system (Furukawa et al., 2003, 2006), which was developed for the detailed characterization of network structure in polymer gels. The SMILS system enables us to scan and measure the light scattering at many different positions in a gel, in order to rigorously determine a time- and space-averaged, i.e. ensemble-averaged, (auto-)correlation function of fluctuating mesh size in the gel. Analysis of the ensemble-averaged function makes it possible to quantitatively characterize the mesh-size distribution of network structure in the gel. Scanning measurement was performed at more than 25 points on a sample to determine ensemble-averaged dynamic structure factor. The determined correlation function was transformed to the distribution function of relaxation time by using numerical inversed Laplace transform calculation. A few peaks of relaxation modes were observed in the distribution function in the present types of organic-inorganic hybrid gels (Naga et al., 2006). Based on the observation of scattering-angle, $q$, dependence of the relaxation modes, all the observed modes usually have $q^{2}$-dependence, which correspond to translational diffusion. In the following, all the results were determined at the scattering angle fixed at $90^{\circ}$. The observed modes, as assigned to the cooperative diffusion of gel network, were used for the determination of radius of mesh (mesh size) $(\xi ; \mathrm{m})$ with Einstein-Stokes formula.

$$
\xi=\frac{16 \pi n^{2} \tau_{R} K_{B} \sin ^{2} \frac{\theta}{2}}{3 \eta \lambda^{2}}
$$

where $n, \tau_{R}, K_{B}, \theta, \eta$, and $\lambda$ are refractive index of toluene or cyclohexane, Ensemble-averaged relaxation time (s), Boltzmann constant $\left(1.38 \times 10^{-23} \mathrm{JK}^{-1}\right)$, scattering angle $\left(90^{\circ}\right)$, viscosity coefficient of solvent at the measured temperature, wave length of incident ray $\left(6.328 \times 10^{-7} \mathrm{~m}\right)$, respectively.

Mechanical properties of the gels were investigated by compression test with Tensilon RTE-1210 (ORIENTEC Co. LTD.). The test samples were cut to $1 \mathrm{~cm}$ cube, and pressed at a rate of $0.5 \mathrm{~mm} / \mathrm{min}$ at room temperature.

\section{Results and Discussion}

\subsection{TMCTS/ $\alpha, \omega$-nonconjugated diene-PS semi-IPN gels In Toluene}

\subsubsection{Effect of PS Concentration on Network Structure of the Semi-IPN Gels}

TMCTS/HD or DD-PS semi-IPN gels were synthesized in the presence of PS1 $\left(M_{\mathrm{n}}=15,000\right)$ in toluene. Effect of PS1 concentration in the semi-IPN gels on the network structure was studied by SMILS. Ensemble-averaged relaxation-time distribution as a function of the relaxation time of the TMCTS/HD-PS semi-IPN gels is shown in Figure 1, and the results are summarized in Table 1. A structure model of TMCTS/HD-PS semi IPN gel is shown in Scheme 2. The peak at the fast relaxation time at around $\tau_{\mathrm{R}}=10^{-6} \mathrm{~s}$ is derived from the mesh of TMCTS/HD network about 1.6-2.1 nm as detected in the gel without PS (Run 1), as shown in Scheme 2 (a). The relaxation peak was detected at around $\tau_{\mathrm{R}}=10^{-2}$ s corresponding to the size of $1100-1300 \mathrm{~nm}$ in the semi-IPN gels containing 2.5 and $5.0 \mathrm{wt} \%$ of PS-1. These relaxation peaks should be derived from the clustered PS chains, as shown in Scheme 2 (c). In the semi-IPN gel containing 2.5 wt $\%$ of PS1, another relaxation peak was observed at $\tau_{\mathrm{R}}=8.8 \times 10^{-5} \mathrm{~s}$ corresponding to the size of $90 \mathrm{~nm}$. The relaxation peak was not detected in the semi-IPN gels containing $5.0 \mathrm{wt} \%$ of PS1. This phenomenon should be derived from lightly clustered PS chains, termed "transition network" concerned with overlapping concentration $\left(\mathrm{C}^{*}\right)$, as shown in Scheme 2 (b) (Simha et al., 1960; de Gennes, 1976, 1979), and we shall return to this subject later.

Figure 2 shows the ensemble-averaged relaxation time distribution as a function of the relaxation time of the TMCTS/DD-PS semi-IPN gels, and the results are summarized in Table 2 . The relaxation peaks observed at around $\tau_{\mathrm{R}}=$ $6-10 \times 10^{-6} \mathrm{~s}$ were derived from the mesh of TMCTS/DD network about 1.1-1.6 nm. The relaxation peaks at around $\tau_{\mathrm{R}}=$ $1.5-2.1 \times 10^{-2} \mathrm{~s}$, which corresponded to the size about $3000 \mathrm{~nm}$, were observed in the semi-IPN gels containing 0.5 and $2.5 \mathrm{wt} \%$ of PS1. The peak was also observed in TMCTS/DD gel without PS, as shown in Figure 2 (a). The relaxation peak should be derived from large defects in the network structure, which was formed by intramolecular cyclization or 
entanglement of long flexible DD, as previously reported. A relaxation peak detected at $\tau_{\mathrm{R}}=2.1 \times 10^{-4} \mathrm{~s}$, which corresponds to the size of $37 \mathrm{~nm}$, in the semi-IPN gels containing $5.0 \mathrm{wt} \%$ of PS-1 should be derived from the transitional network of PS chains as observed in the TMCTS/HD-PS1 semi-IPN gel. The semi-IPN gel containing 5.0 wt $\%$ of PS1 also showed a relaxation peak at $\tau_{\mathrm{R}}=4.3 \times 10^{-3} \mathrm{~s}$. The relaxation peak corresponds to the size of $770 \mathrm{~nm}$, and would be derived from the clustered PS chains.

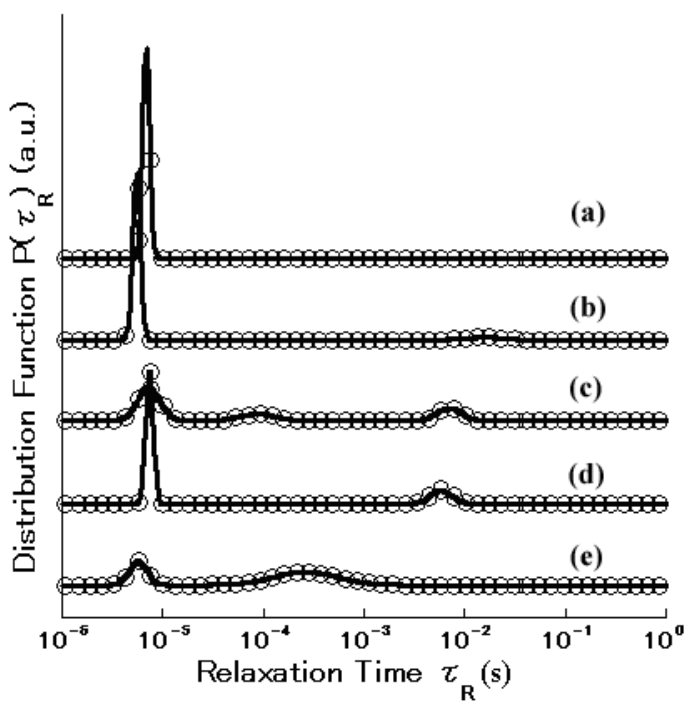

Figure 1. Ensemble-averaged relaxation time distributions as a function of the relaxation time of TMCTS/HD-PS semi-IPN gels in toluene, monomer concentration $=12.0 \mathrm{wt} \%$; (a) without PS, (b) $0.5 \mathrm{wt} \%$ of PS1, (c) $2.5 \mathrm{wt} \%$ of PS1, (d) $5.0 \mathrm{wt} \%$ of PS1, and (e) $0.5 \mathrm{wt} \%$ of PS2.

Table 1. Network structure of TMCTS/HD-PS semi-IPN gel in toluene determined by SMILS ${ }^{\mathrm{a}}$

\begin{tabular}{cccccc}
\hline Run & PS & wt $\%$ & $\tau_{\mathrm{R}} \times 10^{-6}$ & $\sigma$ & $\begin{array}{c}\text { Mesh size } \\
\mathrm{nm}\end{array}$ \\
\hline 1 & & & $\mathrm{~s}$ & & 1.6 \\
2 & --- & 0 & 6.9 & 0.03 & 1.0 \\
3 & PS1 & 0.5 & 8.7 & 0.04 & 1.3 \\
& PS1 & 2.5 & 7.3 & 0.04 & 15.7 \\
& & & 87.9 & 0.19 & 1290 \\
4 & & & 7440 & 0.12 & 2.1 \\
& & & 7.5 & 0.08 & 1070 \\
5 & & 5.0 & 5990 & 0.11 & 1.0 \\
& & & 5.8 & 0.08 & 47.8
\end{tabular}

${ }^{\mathrm{a}}$ Monomer concentration; TMCTS+HD $=12.0 \mathrm{wt} \%{ }^{\mathrm{b}}$ Relaxation time. ${ }^{\mathrm{c}}$ Standard deviation of a peak of the ensemble-averaged relaxation time distribution. 


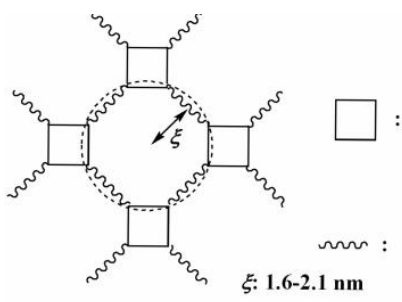

(a) Mesh of TMCTS/HD

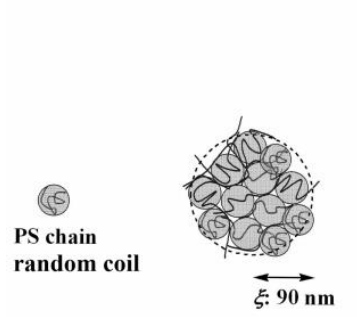

(b) Transition network
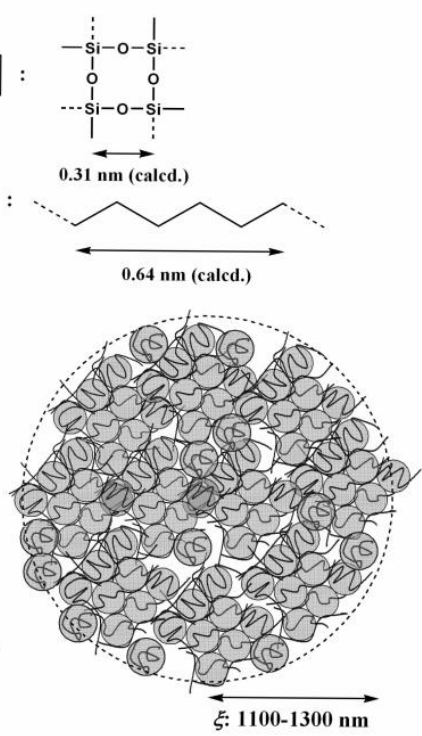

(c) Clustered PS chains

Scheme 2. Structure models of TMCTS/HD mesh (a) and PS chains in the semi-IPN gel; transition network (b), clustered structure (c).

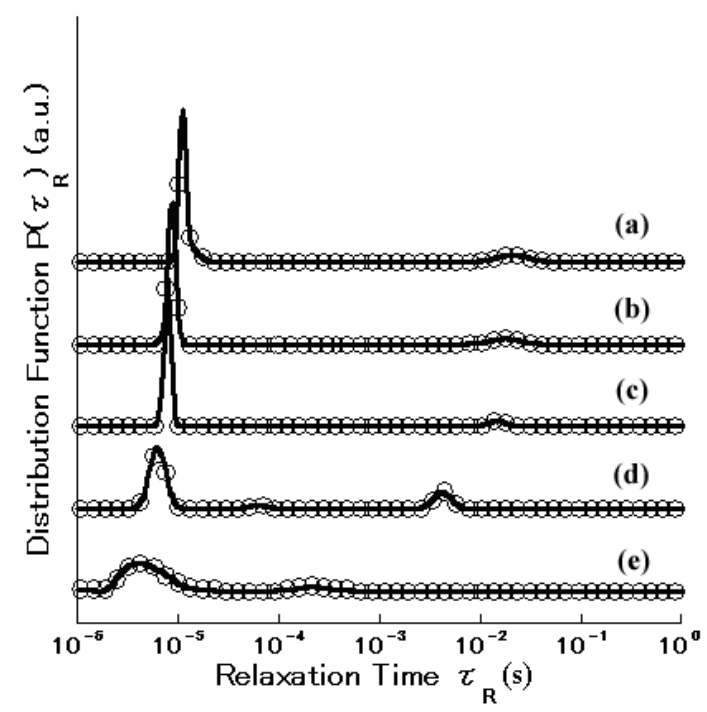

Figure 2. Ensemble-averaged relaxation time distributions as a function of the relaxation time of TMCTS/DD-PS semi-IPN gels in toluene, monomer concentration $=10.0 \mathrm{wt} \%$; (a) without PS, (b) $0.5 \mathrm{wt} \%$ of PS1, (c) $2.5 \mathrm{wt} \%$ of PS1, (d) $5.0 \mathrm{wt} \%$ of PS1, and (e) $0.5 \mathrm{wt} \%$ of PS2. 
Table 2. Network structure of TMCTS/DD-PS semi-IPN gel in toluene determined by SMILS ${ }^{\text {a }}$

\begin{tabular}{|c|c|c|c|c|c|}
\hline Run & PS & $\mathrm{wt} \%$ & $\begin{array}{c}\tau_{\mathrm{R}}^{\mathrm{b}} \times 10^{-6} \\
\mathrm{~s}\end{array}$ & $\sigma^{c}$ & $\begin{array}{c}\text { Mesh size } \\
\mathrm{nm}\end{array}$ \\
\hline \multirow[t]{2}{*}{6} & --- & 0 & 11.1 & 0.04 & 2.0 \\
\hline & & & 21300 & 0.04 & 3810 \\
\hline \multirow[t]{2}{*}{7} & PS1 & 0.5 & 8.7 & 0.04 & 1.6 \\
\hline & & & 17900 & 0.14 & 3190 \\
\hline \multirow[t]{2}{*}{8} & PS1 & 2.5 & 7.9 & 0.03 & 1.4 \\
\hline & & & 15000 & 0.09 & 2670 \\
\hline \multirow[t]{3}{*}{9} & PS1 & 5.0 & 6.3 & 0.08 & 1.1 \\
\hline & & & 34.3 & 0.09 & 11.5 \\
\hline & & & 4310 & 0.09 & 769 \\
\hline \multirow[t]{2}{*}{10} & PS2 & 0.5 & 4.6 & 0.20 & 0.8 \\
\hline & & & 225 & 0.14 & 40.1 \\
\hline
\end{tabular}

${ }^{\mathrm{a}}$ Monomer concentration; TMCTS+DD $=10.0 \mathrm{wt} \%$. ${ }^{\mathrm{b}}$ Relaxation time. ${ }^{\mathrm{c}}$ Standard deviation of a peak of the ensemble-averaged relaxation time distribution.

\subsubsection{Effect of Molecular Weight of PS on the Network Structure}

Effect of molecular weight of PS on the network structure of the semi-IPN gel was studied by SMILS. We prepared the semi-IPN gels containing $0.5 \mathrm{wt} \%$ of high molecular weight PS, PS2 $M_{\mathrm{n}}=69,000$ (Runs 19 and 24). The ensemble-averaged relaxation time distributions as a function of the relaxation time of TMCTS/HD-PS2 and TMCTS/DD-PS2 semi-IPN gels are illustrated in Figure 1 (e) and Figure 2 (e), respectively. In the case of the TMCTS/HD-PS2 semi-IPN gel, a broad relaxation peak derived from the transitional network of clustered PS chains was observed at around $\tau_{\mathrm{R}}=2.7 \times 10^{-4} \mathrm{~s}$ corresponding to the size of $48 \mathrm{~nm}$. Furthermore, the relaxation peak derived from the mesh of TMCTS/HD network at around $\tau_{\mathrm{R}}=5.8 \times 10^{-6} \mathrm{~s}$, corresponding to the size of $1.0 \mathrm{~nm}$, became broad in comparison with that of the semi-IPN gel containing low molecular weight PS1, as shown in Figure 1 (b). These phenomena were also observed in the relaxation time distributions as a function of the relaxation time of the TMCTS/DD-PS2 semi-IPN gels, as shown in Figure 2 (e). In addition, the mesh size of the TMCTS/DD network in the semi-IPN gel containing PS2 $(0.83 \mathrm{~nm})$ was smaller than that of the corresponding gel containing PS1 $(1.55 \mathrm{~nm})$.

We can then go on to consider the network model of the semi-IPN gels in toluene base on the observations of the relaxation peaks of both the TMCTS/ $\alpha, \omega$-nonconjugated diene and PS chains. The relaxation peaks derived from the transitional network or the clustered PS chains were detected in both the TMCTS/HD-PS and TMCTS/DD-PS semi-IPN gels by SMILS analysis. The transition network would be formed by lightly clustered PS chains (Scheme 2 (b)) when the concentration of PS is around the overlapping concentration $\left(\mathrm{C}^{*}\right)$. This state should correspond to the TMCTS/HD-PS semi-IPN gels containing $2.5 \mathrm{wt} \%$ of PS1 and $0.5 \mathrm{wt} \%$ of PS2, Figures 1 (c) and (e), and the TMCTS/DD-PS semi-IPN gels containing $5.0 \mathrm{wt} \%$ of PS1 and $0.5 \mathrm{wt} \%$ of PS2, Figures 2 (d) and (e). Further aggregation of the transition networks would form larger cluster (Scheme 2 (c)), as detected in Figure 1 (c) and Figure 2 (d). In the case of the TMCTS/HD-PS semi-IPN gel, the relaxation peaks derived from the transition network and clustered PS chains were detected in the semi-IPN gels containing low concentration of PS1 $(2.5 \mathrm{wt} \%)$. Whereas, these relaxation peaks were not detected in the corresponding TMCTS/DD-PS semi-IPN gel containing $2.5 \mathrm{wt} \%$ of PS1. The difference would indicate that the PS chains may cluster easier in the semi-IPN gel with HD than in that with DD.

Clear correlation was not observed between the mesh size derived from TMCTS/HD network and the concentration or molecular weight of PS in the semi-IPN gels. On the other hand, the mesh size derived from TMCTS/DD network slightly decreased with increasing of the PS concentration. Increase of the molecular weight of PS decreased the mesh size of the TMCTS/DD network in the same way. These results can be explained by a following model, as shown in Scheme 3. Some part of PS chains would penetrate into the TMCTS/DD network or entangle with the TMCTS/DD mesh during formation process of the semi-IPN gel. After the gelation, the aggregation of PS chains may decrease the mesh size of the TMCTS/DD network. The aggregation would be accelerated by increasing of the concentration and molecular weight of PS. 


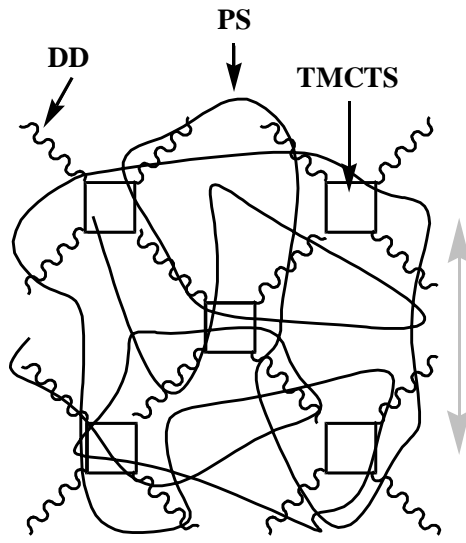

low PS concentration

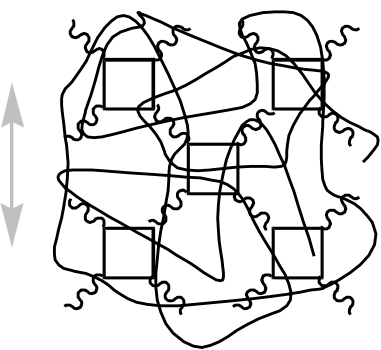

high PS concentration

Scheme 3. Plausible model of TMCTS/DD-PS semi-IPN gel in toluene

\subsection{TMCTS/ $\alpha$, $\omega$-nonconjugated diene-PS semi-IPN gels in Cyclohexane}

\subsubsection{Effect of Temperature on Network Structure of Semi-IPN Gels}

The TMCTS/HD or DD-PS semi-IPN gels containing $0.1 \mathrm{wt} \%$ of PS3 were also synthesized in cyclohexane, which is a poor solvent for PS. PS3 was used in this experiment, because the sample showed UCST at $34^{\circ} \mathrm{C}$ in cyclohexane. The UCST is a little higher than room temperature and convenient to investigate the effect of the temperature on the network structure of the semi-IPN gels by SMILS. The TMCTS/HD-PS3 semi-IPN gel was prepared at $40^{\circ} \mathrm{C}$, and the network structure of the resulting semi-IPN gel was traced with SMILS on the cooling process. The ensemble-averaged relaxation-time distributions as a function of the relaxation time are shown in Figure 3, and the results are summarized in Table 3. The relationship between the temperature and mesh size of the TMCTS/HD-PS3 semi-IPN gel is shown in Figure 4. In the SMILS analysis of the TMCTS/HD-PS3 semi-IPN gel at $40^{\circ} \mathrm{C}$, a relaxation peak was detected at $\tau_{\mathrm{R}}=$ $5.2 \times 10^{-6}$ s corresponding to $1.2 \mathrm{~nm}$ of size. This size was close to that of the mesh size of the TMCTS/HD gel (1.1 nm) prepared without PS. The size derived from the TMCTS/HD mesh increased with decreasing of the temperature over the UCST $\left(>34^{\circ} \mathrm{C}\right)$. There was a rapid decrease in the mesh size below the UCST. Another relaxation peak was detected at around $\tau_{\mathrm{R}}=10^{-4}-10^{-3} \mathrm{~s}$ corresponding to the size of 900-2300 $\mathrm{nm}$ derived from the large clustered PS chains. The size was almost fixed about 2000-2300 nm independent of the temperature over the UCST (40-34 $\left.{ }^{\circ} \mathrm{C}\right)$. The relaxation peak also rapidly sifted to short time at around $\tau_{\mathrm{R}}=10^{-4}-10^{-3} \mathrm{~s}$ corresponding to the size of 930-980 nm, and its intensity drastically decreased below the $\operatorname{UCST}\left(<32^{\circ} \mathrm{C}\right)$.

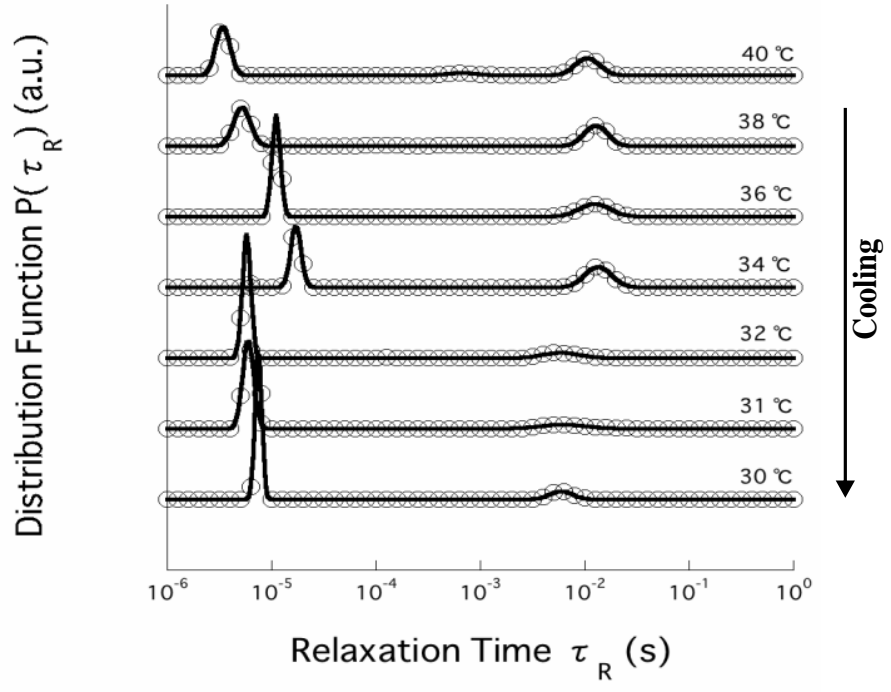

Figure 3 Ensemble-averaged relaxation-time distributions as a function of the relaxation time on the cooling process of TMCTS/HD-PS3 semi-IPN gel prepared at $40^{\circ} \mathrm{C}$ on the cooling process. 
Table 3. Network structure of TMCTS/HD-PS3 semi-IPN gel in cyclohexane determined by SMILS on the cooling process $^{\mathrm{a}}$

\begin{tabular}{|c|c|c|c|c|}
\hline Run & $\begin{array}{c}\text { Temperature } \\
{ }^{\circ} \mathrm{C}\end{array}$ & $\tau_{\mathrm{R}}^{\mathrm{b}} \times 10^{-6}$ & $\sigma^{c}$ & $\begin{array}{c}\text { Mesh size } \\
\mathrm{nm}\end{array}$ \\
\hline & & $\mathrm{s}$ & & \\
\hline \multirow[t]{2}{*}{11} & 40 & 3.4 & 0.07 & 0.6 \\
\hline & & 10600 & 0.12 & 2000 \\
\hline \multirow[t]{2}{*}{12} & 38 & 5.2 & 0.08 & 1.0 \\
\hline & & 12800 & 0.11 & 2340 \\
\hline \multirow[t]{2}{*}{13} & 36 & 11.1 & 0.04 & 2.0 \\
\hline & & 12400 & 0.15 & 2180 \\
\hline \multirow[t]{2}{*}{14} & 34 & 17.1 & 0.05 & 2.9 \\
\hline & & 13400 & 0.13 & 2270 \\
\hline \multirow[t]{2}{*}{15} & 32 & 5.8 & 0.04 & 0.9 \\
\hline & & 5870 & 0.17 & 960 \\
\hline \multirow[t]{2}{*}{16} & 31 & 6.0 & 0.05 & 1.0 \\
\hline & & 6130 & 0.23 & 982 \\
\hline \multirow[t]{2}{*}{17} & 30 & 7.5 & 0.03 & 1.2 \\
\hline & & 5920 & 0.11 & 928 \\
\hline
\end{tabular}

${ }^{\mathrm{a}} \mathrm{PS} 3=0.1 \mathrm{wt} \%$, Monomer concentration; TMCTS+HD $=12.0 \mathrm{wt} \% \cdot{ }^{\mathrm{b}}$ Relaxation time. ${ }^{\mathrm{c}}$ Standard deviation of a peak of the ensemble-averaged relaxation time distribution.

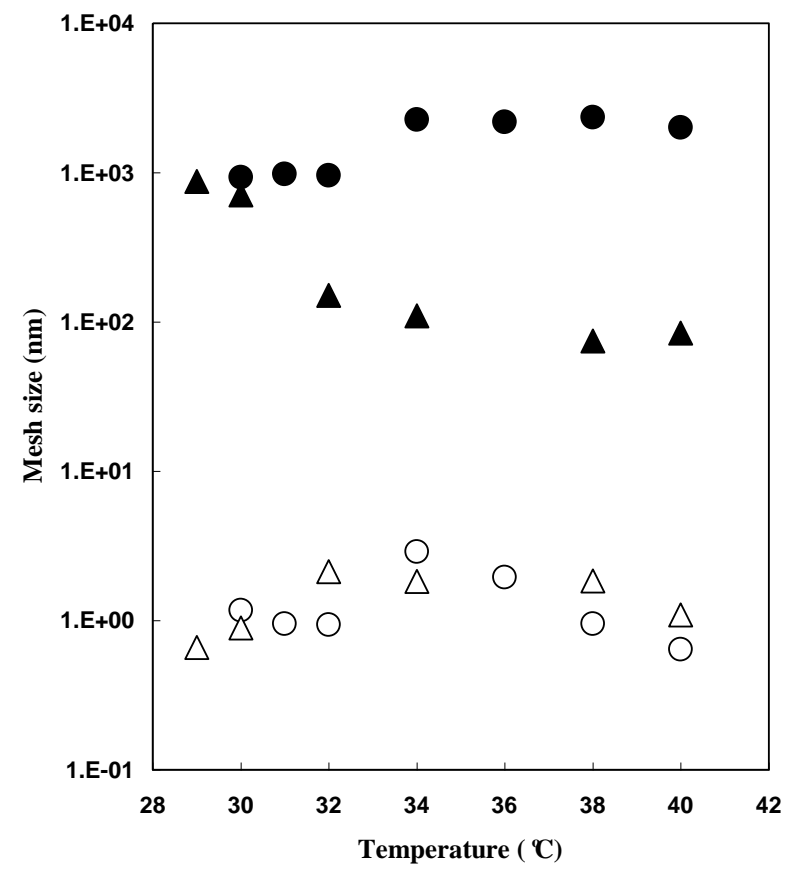

Figure 4 Temperature dependence of mesh size of TMCTS/HD-PS3 semi-IPN gel (circle) and TMCTS/DD-PS3 semi-IPN gel (triangle) on the cooling process.

The TMCTS/DD-PS3 semi-IPN gel was also prepared at $40^{\circ} \mathrm{C}$, and the network structure of the semi-IPN gel was investigated with SMILS on the cooling process. The ensemble-averaged relaxation-time distributions as a function of the relaxation time are shown in Figure 5, and the results are summarized in Table 4. The relationship between the temperature and mesh size of the TMCTS/DD-PS3 semi-IPN gel is also plotted in Figure 4. In the ensemble-averaged relaxation-time distribution acquired at $40^{\circ} \mathrm{C}$, a relaxation peak was detected at $\tau_{\mathrm{R}}=5.8 \times 10^{-6} \mathrm{~s}$ corresponding to $1.1 \mathrm{~nm}$ 
derived from the TMCTS/DD mesh. The mesh size slightly increased with decreasing of the temperature over the UCST $\left(34^{\circ} \mathrm{C}\right)$, whereas it returned to the original size below the UCST. Another relaxation peak was detected at slow relaxation time at around $\tau_{\mathrm{R}}=10^{-4} \mathrm{~s}$ corresponding to the size about $80 \mathrm{~nm}$ derived from the transition network over the UCST, and the size increased to $710-870 \mathrm{~nm}$ below the UCST $\left(30,29^{\circ} \mathrm{C}\right)$. The result indicates that aggregation of the transition network would form the clustered PS chains under the UCST.

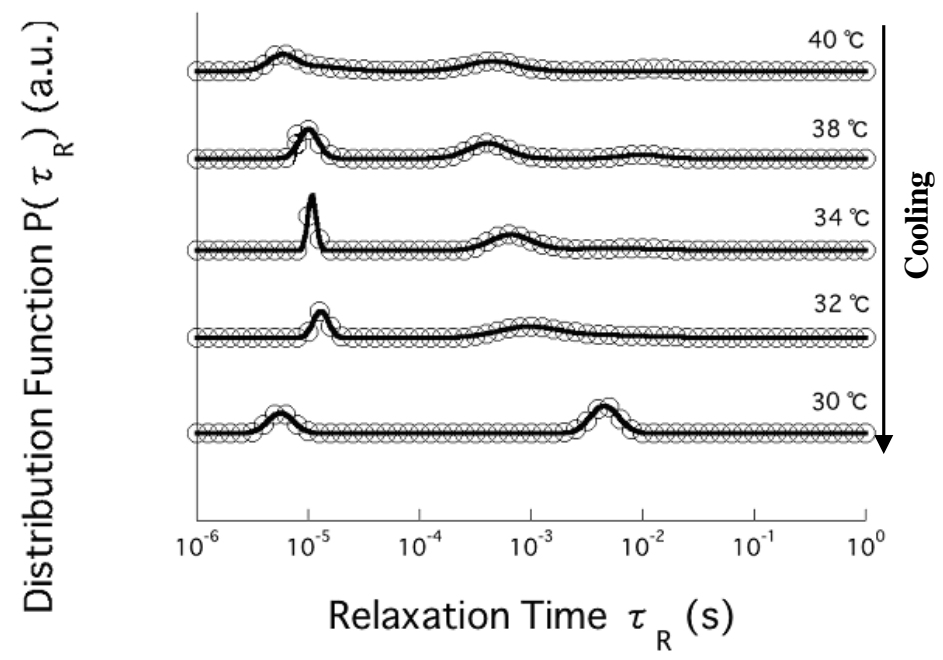

Figure 5. Ensemble-averaged relaxation-time distributions as a function of the relaxation time of TMCTS/DD-PS3 semi-IPN gel prepared at $40^{\circ} \mathrm{C}$ on the cooling process.

Table 4. Network structure of TMCTS/DD-PS3 semi-IPN gel in cyclohexane on the cooling process ${ }^{\mathrm{a}}$

\begin{tabular}{|c|c|c|c|c|}
\hline Run & $\begin{array}{c}\text { Temperature } \\
{ }^{\circ} \mathrm{C}\end{array}$ & $\begin{array}{c}\tau_{\mathrm{R}}^{\mathrm{b}} \times 10^{-6} \\
\mathrm{~s}\end{array}$ & $\sigma^{c}$ & $\begin{array}{c}\text { Mesh size } \\
\mathrm{nm}\end{array}$ \\
\hline \multirow[t]{2}{*}{18} & 40 & 5.8 & 0.07 & 1.1 \\
\hline & & 446 & 0.23 & 84.3 \\
\hline \multirow[t]{2}{*}{19} & 38 & 10.1 & 0.09 & 1.8 \\
\hline & & 409 & 0.18 & 74.6 \\
\hline \multirow[t]{2}{*}{20} & 34 & 10.8 & 0.04 & 1.8 \\
\hline & & 650 & 0.19 & 110 \\
\hline \multirow[t]{2}{*}{21} & 32 & 13.0 & 0.07 & 2.1 \\
\hline & & 920 & 0.27 & 150 \\
\hline \multirow[t]{2}{*}{22} & 30 & 5.7 & 0.11 & 0.9 \\
\hline & & 4530 & 0.13 & 711 \\
\hline \multirow[t]{2}{*}{23} & 29 & 4.3 & 0.07 & 0.7 \\
\hline & & 5654 & 0.07 & 871 \\
\hline
\end{tabular}

${ }^{\mathrm{a}} \mathrm{PS} 3=0.1 \mathrm{wt} \%$, Monomer concentration; TMCTS+DD $=10.0 \mathrm{wt} \% \cdot{ }^{\mathrm{b}}$ Relaxation time. ${ }^{\mathrm{c}}$ Standard deviation of a peak of the ensemble-averaged relaxation time distribution.

We are now ready to consider the effect of the temperature on the network structure of the semi-IPN gels on the cooling process. In the case of the TMCTS/HD-PS3 semi-IPN gel, a relaxation peak corresponding to the size about $2000 \mathrm{~nm}$ derived from the large clustered PS chains was detected over the UCST. When the temperature dropped below the UCST, the semi-IPN gel became muddy due to the insolubility of the PS chains, and which would gather with the TMCTS/HD network. A part of PS chains would form clusters about $1000 \mathrm{~nm}$.

In the case of TMCTS/DD-PS3 semi-IPN gel, the clustered PS chains formed the transition network about $100 \mathrm{~nm}$ over the UCST. The transition networks would gradually aggregate with decreasing the temperature over the UCST. When 
the temperature dropped below the UCST, the size drastically increased due to the acceleration of the aggregation of the transition networks to form the clustered PS chains.

The size of small mesh derived from both the TMCTS/HD and TMCTS/DD networks slightly increased with decreasing of the temperature over the UCST. When the temperature dropped below the UCST, the mesh size returned to almost the original size at prepared temperature $\left(40^{\circ} \mathrm{C}\right)$. The temperature dependence of the mesh size was observed only in the semi-IPN gels. Although the mechanism has not cleared yet, the interaction between the network and PS chains should induce the phenomena.

\subsection{Mechanical Properties of the Semi-IPN gels}

The mechanical properties of the semi-IPN gels prepared in toluene were investigated by the compression test. Stress-strain curves of TVMCTS/HD, DD-PS1 gels, and TVMCTS/HD, DD gels for reference, are shown in Figure 6. Young's modulus, breaking stress, and breaking point, of the prepared gels are summarized in Table 5. Although all the gels were fragile, the semi-IPN gels showed higher Young's modulus and breaking stress than the gels without PS1. The PS in the network structure of the gels should enhance the mechanical properties of the gels.

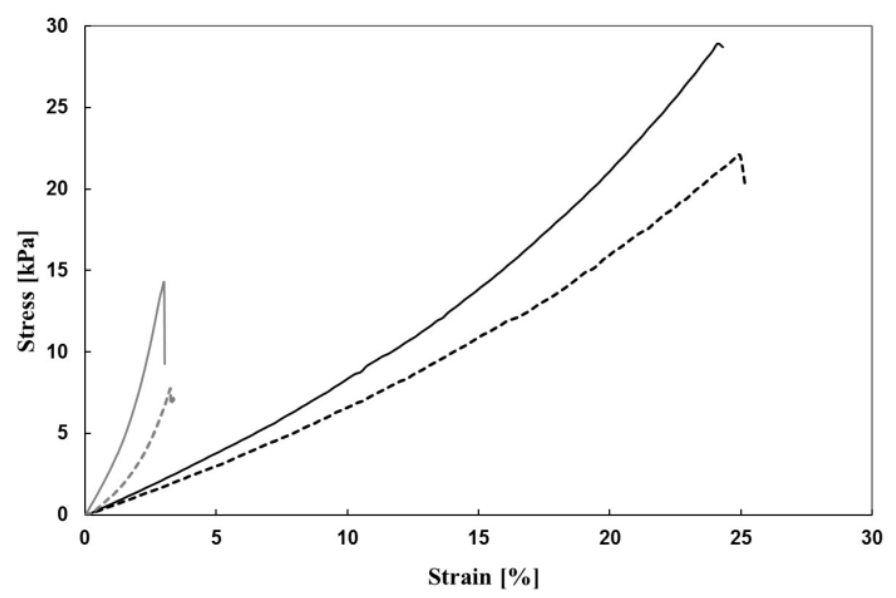

Figure 3 Stress-strain curves of TMCTS/HD gel (gray dot line), TMCTD/HD-PS1 semi-IPN (gray line), TMCTS/DD gel (black dot line), and TMCTS/DD-PS1 semi-IPN (black line) in toluene, monomer concentration $=10 \mathrm{wt} \%$ (TMCTS/HD), $12 \mathrm{wt} \%$ (TMCTS/DD).

Table 5. Compression test of TMCTS-HD, DD gels, monomer concentration $12 \mathrm{wt} \%$.

\begin{tabular}{cccccc}
\hline Run & Network & $\begin{array}{c}\text { PS1 } \\
{[\mathrm{wt} \%]}\end{array}$ & $\begin{array}{c}\text { Young's modulus } \\
{[\mathrm{kPa}]}\end{array}$ & $\begin{array}{c}\text { Breaking stress } \\
{[\mathrm{kPa}]}\end{array}$ & $\begin{array}{c}\text { Breaking point } \\
{[\%]}\end{array}$ \\
1 & TMCTS/HD & 0 & 104.8 & 7.8 & 3.3 \\
4 & TMCTS/HD & 5 & 250.1 & 14.3 & 3.0 \\
6 & TMCTS/DD & 0 & 45.1 & 22.1 & 25.2 \\
9 & TMCTS/DD & 5 & 91.2 & 28.9 & 24.3 \\
\hline
\end{tabular}

\section{Conclusion}

The TMCTS/HD or DD-PS semi-IPN gels were successfully synthesized by means of a hydrosilylation reaction using a Pt catalyst, and network structure of the semi-IPN gels was quantitatively investigated by SMILS. Features of the solvent and molecular length of the $\alpha, \omega$-nonconjugated dienes strongly affected the network structures of the resulting semi-IPN gels. The following phenomena were cleared by the structure analysis of the semi-IPN gels by SMILS. In the case of the semi-IPN gels in toluene, the transition network or cluster of PS chains was formed in the semi-IPN gels containing high concentration or high molecular weight PS. These structures derived from PS chains were easily formable in the semi-IPN gels with DD. In the case of the semi-IPN gels in cyclohexane, the transition networks or clustered PS chains were detected in the semi-IPN gels containing low concentration, $0.1 \mathrm{wt} \%$, of PS chains. In the semi-IPN gels with HD, a relaxation peak derived large clustered PS chains about 2000-3000 nm size was detected in the SMILS analysis over the UCST. On the other hand, the SMILS analysis of the semi-IPN gels with DD indicated that the existence of the transition networks about $75-150 \mathrm{~nm}$ size. The PS chains in the semi-IPN gels in cyclohexane were educed below the UCST, and formed relatively small clustered structure about 710-870 nm. These results indicate that PS chains can be aggregated easier in the semi-IPN gels with HD than those with DD. The PS chains would penetrate into the TMCTS/DD network or entangle with network in the TMCTS/DD-PS semi-IPN gel due to the large mesh size 
of the TMCTS/DD gel, as previously reported. The PS chains in the semi-IPN gels were effective to improve the mechanical properties of the hybrid gels.

The fundamental studies of semi-IPN gel composed of multi-functional siloxane and $\alpha, \omega$-nonconjugated dienes by means of a hydrosilylation reaction in the presence of a linear polymer should be useful for the application of the functionalized organic-inorganic hybrid gel. Developments of the organic-inorganic hybrid semi-IPN gels with photo-function, electrical-function, or excellent mechanical property are now proceeding, and the results will be reported elsewhere.

\section{References}

Choi, J., Yee, A. F., \& Laine, R. M. (2003). Organic/Inorganic Hybrid Composites from Cubic Silsesquioxanes. Epoxy Resins of Octa(dimethylsiloxyethylcyclohexylepoxide) Silsesquioxane. Macromolecules, 36, 5666-5682. http://pubs.acs.org/doi/abs/10.1021/ma030172r

de Gennes, P. G. (1976). Dynamics of Entangled Polymer Solutions. I. The Rouse Model. Macromolecules, 9, 587-593. http://pubs.acs.org/doi/abs/10.1021/ma60052a011

de Gennes, P. G. (1976). Dynamics of Entangled Polymer Solutions. II. Inclusion of Hydrodynamic Interactions. Macromolecules, 9, 594-598. http://pubs.acs.org/doi/abs/10.1021/ma60052a012

de Gennes, P. G. (1979). Scaling Concepts in Polymer Physics, Ithaca, NY, Cornell University Press. http://www.cornellpress.cornell.edu/book/?GCOI=80140100785990

Furukawa, H., Horie K., Nozaki, R., \& Okada, M. (2003). Swelling-induced modulation of static and dynamic fluctuations in polyacrylamide gels observed by scanning microscopic light scattering. Phys. Rev. E, Statistical, Nonlinear, and Soft Matter Phys. 68, 031406-1. http://journals.aps.org/pre/abstract/10.1103/PhysRevE.68.031406

Furukawa, H., Kobayashi, M., Miyashita, Y., \& Horie, K. (2006). End-Crosslinking Gelation of Poly(amide acid) Gels studied with Scanning Microscopic Light Scattering. High Performance Polymers, 18, 837-847. http://hip.sagepub.com/content/18/5/837.abstract

Galiastsatos, V., Subramanian, P. R., \& Klein-Castner, L. (2001). Designing heterogeneity into bimodal elastomeric PDMS networks. Macromol. Symp., 97-104. http://onlinelibrary.wiley.com/doi/10.1002/1521-3900(200106)171:1\%3C97::AID-MASY97\%3E3.0.CO;2-1/abstra ct

Gong, J. P., Katsuyama, Y., Kurokawa, T., \& Osada, Y. (2003). Double-Network Hydrogels with Extremely High Mechanical Strength. Adv. Mater., 15, 1155-1158. http://onlinelibrary.wiley.com/doi/10.1002/adma.200304907/full

Kim, K. M., \& Chujo, Y. (2003). Organic-inorganic hybrid gels having functionalized silsesquioxanes. J. Mater. Chem., 13, 1384-1391. http://pubs.rsc.org/en/content/articlelanding/2003/jm/b211030j\#!divAbstract

Krishnamoorti, R., Vaia, R. A., \& Giannelis, E. P. (1996). Structure and Dynamics of Polymer-Layered Silicate Nanocomposites. Chemistry of Materials, 8, 1728-1734. http://pubs.acs.org/doi/abs/10.1021/cm960127g

Laine, R. M., Zhang, C., Sellinger, A., \& Viculis, L. (1998). Polyfunctional cubic silsesquioxanes as building blocks for organic/inorganic hybrids. Appl. Organomet. Chem., 12, 715-723. http://onlinelibrary.wiley.com/doi/10.1002/(SICI)1099-0739(199810/11)12:10/11\%3C715::AID-AOC778\%3E3.0. $\mathrm{CO} ; 2-0 / \mathrm{abstract}$

Landry, C. J. T., Coltrain, B. K., Landry, M. R., \& Long V. K. (1993). Poly(vinyl acetate)/silica-filled materials: material properties of in situ vs fumed silica particles. Macromolecules, 26, 3702-3712. http://pubs.acs.org/doi/abs/10.1021/ma00066a032?journalCode=mamobx

Mark, J. E., Lee, C., \& Bianconi P. A. (1995). Hybrid Organic-Inorganic Composites. ACS Symposium Series 585. http://pubs.acs.org/isbn/9780841231481

Mascia, L. (1995). Developments in organic-inorganic polymeric hybrids: Ceramers. Trends in Polymer Science, 3, $61-66$.

Michalczyk, M. J., Farneth, W. E., \& Vega, A. J. (1993). High temperature stabilization of crosslinked siloxanes glasses. Chem. Mater., 5, 1687-1689. http://pubs.acs.org/doi/abs/10.1021/cm00036a001

Na, Y. H., Kurokawa, T., Katsuyama, Y., Tsukeshiba, H., Gong, J. P., Osada, Y., Okabe, S., Karino, T., \& Shibayama, M. (2004). Structural Characteristics of Double Network Gels with Extremely High Mechanical Strength. Macromolecules, 37, 5370-5374. http://pubs.acs.org/doi/abs/10.1021/ma049506i

Naga, N., Oda, E., Toyota, A., \& Furukawa, H. (2007). Mesh Size Control of Organic-Inorganic Hybrid Gels by Means 
of a Hydrosilylation Co-Gelation of Siloxane or Silsesquioxane and $\boldsymbol{\alpha}$, $\boldsymbol{\omega}$-Non-Conjugated Dienes. Macromol. Chem. Phys., 208, 2331-2338. http://onlinelibrary.wiley.com/doi/10.1002/macp.200700184/abstract

Naga, N., Oda, E., Toyota, A., Horie, K., \& Furukawa, H. (2006). Tailored Synthesis and Fundamental Characterization of Organic-Inorganic Hybrid Gels by Means of a Hydrosilylation Reaction. Macromol. Chem. Phys., 207, 627-635. http://onlinelibrary.wiley.com/doi/10.1002/macp.200500501/abstract

Nakayama, A., Kakugo, A,, Gong, J. P., Osada, Y., Takai, M., \& Erata, T. (2004). High Mechanical Strength Double-Network Hydrogel with Bacterial Cellulose. Adv. Func. Mater, 14, 1124-1128. http://onlinelibrary.wiley.com/doi/10.1002/adfm.200305197/abstract

Novak, B. M. (1993). Hybrid Nanocomposite Materials-between inorganic glasses and organic polymers. Adv. Mater., 5 , 422-433. http://onlinelibrary.wiley.com/doi/10.1002/adma.19930050603/pdf

Pan, G., Mark, J. E., \& Schaefer D. W. (2003). Synthesis and characterization of fillers of controlled structure based on polyhedral oligomeric silsesquioxane cages and their use in reinforcing siloxane elastomers. J. Polym. Sci. Part B: Polym. Phys., 41, 3314-3323. http://onlinelibrary.wiley.com/doi/10.1002/polb.10695/full

Pinho, R. O., Radovanovic, E., Torriani, I. L., \& Yoshida, I. V. P. (2004). Hybrid materials derived from divinylbenzene $\begin{array}{lllll}\text { and cyclic } & \text { siloxane. } & \text { Eur. } & \text { Polym. } & \text { J., }\end{array}$ http://www.sciencedirect.com/science/article/pii/S0014305703002489

Provatas, A., \& Matisons, J. G. (1997). Silsesquioxanes: synthesis and applications. Trends in Polymer Science, 5, 327-332. http://cat.inist.fr/?aModele $=$ afficheN\&cpsidt=2828673

Redondo, S. U. A., Radovanovic, E., Torriani, I. L., \& Yoshida, I. V. P. (2001). Polycyclic silicone membranes. Synthesis, characterization and permeability evaluation. Polymer, 42, 1319-1327. http://www.sciencedirect.com/science/article/pii/S0032386100005206

Saegusa. T., \& Chujo. Y. (1991). Macromolecular engineering on the basis of the polymerization of 2-oxazolines. Makromol. Chem. Macromol. $\quad$ Symp., $\quad 51$, 1-10. http://onlinelibrary.wiley.com/doi/10.1002/masy.19910510103/abstract;jsessionid=4332BBF608B5946C6B7DE19 0327D4E1D.f02t03

Simha, R., \& Zakin, J. L. (1960). Compression of Flexible Chain Molecules in Solution. J. Chem. Phys., 33, 1791. http://scitation.aip.org/content/aip/journal/jcp/33/6/10.1063/1.1731504

Su, R. Q., Müller, T. E., Procházka, J., \& Lercher J. (2002). A New Type of Low-x Dielectric Films Based on Polysilsesquioxanes. $\quad A d v$. Mater, $\quad 14, \quad 1369-1373$. http://onlinelibrary.wiley.com/doi/10.1002/1521-4095(20021002)14:19\%3C1369::AID-ADMA1369\%3E3.0.CO;2I/epdf

Tominaga, T., Tirumala, V. R., Lin E. K., Gong, J. P., Furukawa, H., Osada, Y., \& Wu, W. L.(2007). The molecular origin of enhanced toughness in double-network hydrogels: A neutron scattering study. Polymer, 48, 7449-7454. http://www.sciencedirect.com/science/article/pii/S0032386107009858

Tsumura, M., \& Iwahara, T. (1999). Synthesis and Properties of Crosslinked Polycarbosilanes by Hydrosilylation Polymerization. Polymer J., 31, 452-457. http://www.nature.com/pj/journal/v31/n5/abs/pj199985a.html

Tsumura, M., \& Iwahara, T. (2000). Crosslinked Polycarbosilanes. Synthesis and Properties. Polymer J., 32, 567-573. http://www.nature.com/pj/journal/v32/n7/abs/pj2000104a.html

Tsumura, M., \& Iwahara, T. (2000). Silicon-based materials prepared by IPN formation and their properties. J. Appl. Polym. $\quad$ Sci., $\quad 78, \quad 724-731$. http://onlinelibrary.wiley.com/doi/10.1002/1097-4628(20001024)78:4\%3C724::AID-APP50\%3E3.0.CO;2-9/abstra ct

Tsumura, M., Ando, K., Kotani, J., Hiraishi, M., \& Iwahara, T. (1998). Silicon-Based Interpenetrating Polymer Networks (IPNs): Synthesis and Properties, Macromolecules, 31, 2716-2723. http://pubs.acs.org/doi/abs/10.1021/ma971308m

Weimer, M. W., Chen, H., \& Giannelis, E. P., Sogah, D. Y. (1999). Direct Synthesis of Dispersed Nanocomposites by in Situ Living Free Radical Polymerization Using a Silicate-Anchored Initiator. J. Am. Chem. Soc., 121, 1615-1616. http://pubs.acs.org/doi/abs/10.1021/ja983751y

Yamada, N., Yoshinaga, I., \& Katayama, S.(1997). Synthesis and dynamic mechanical behaviour ofinorganic-organic hybrids containing various inorganiccomponents. J. Mater. Chem., 7, 1491-1495. http://pubs.rsc.org/en/Content/ArticleLanding/1997/JM/a700793k\#!divAbstract 
Yasuda, K., Gong, J. P., Katsuyama, Y., Nakayama, A., Tanabe, Y., Kondo, E., Ueno, M., \& Osada, Y. (2005). Biomechanical properties of high-toughness double network hydrogels. Biomaterials, 26, 4468-4475. http://www.sciencedirect.com/science/article/pii/S0142961204010038

Zhang, C., Babonneau, F., Bonhomme, C., Laine, R. M., Soles, C. L., Hristov, H. A., \& Yee, A. F. (1998). Highly Porous Polyhedral Silsesquioxane Polymers. Synthesis and Characterization. J. Am. Chem. Soc., 120, 8380-8391. http://pubs.acs.org/doi/abs/10.1021/ja9808853

\section{Copyrights}

Copyright for this article is retained by the author(s), with first publication rights granted to the journal.

This is an open-access article distributed under the terms and conditions of the Creative Commons Attribution license (http://creativecommons.org/licenses/by/3.0/). 